\title{
Stabilization of the Fractional-Order Chua Chaotic Circuit via the Caputo Derivative of a Single Input
}

\author{
Chunde Yang, ${ }^{1}$ Hao Cai, ${ }^{2}$ and Ping Zhou ${ }^{1,2}$ \\ ${ }^{1}$ Center of System Theory and Its Applications, Chongqing University of Posts and Telecommunications, Chongqing 400065, China \\ ${ }^{2}$ Key Laboratory of Network Control and Intelligent Instrument of Ministry of Education, \\ Chongqing University of Posts and Telecommunications, Chongqing 400065, China
}

Correspondence should be addressed to Ping Zhou; zhouping@cqupt.edu.cn

Received 8 September 2015; Accepted 8 November 2015

Academic Editor: Chris Goodrich

Copyright (C) 2016 Chunde Yang et al. This is an open access article distributed under the Creative Commons Attribution License, which permits unrestricted use, distribution, and reproduction in any medium, provided the original work is properly cited.

\begin{abstract}
A modified fractional-order Chua chaotic circuit is proposed in this paper, and the chaotic attractor is obtained for $q=0.98$. Based on the Mittag-Leffler function in two parameters and Gronwall's Lemma, two control schemes are proposed to stabilize the modified fractional-order Chua chaotic system via the Caputo derivative of a single input. The numerical simulation shows the validity and feasibility of the control scheme.
\end{abstract}

\section{Introduction}

Chaotic phenomena have been observed in many physical systems, such as Chua's circuit and its fractional-order model $[1,2]$, the brushless DC motor [3], the microelectromechanical system [4], and the fractional-order gyroscopes [5]. Sometimes, chaos is undesirable in the field of mechanical engineering and power transmission, for example, in motor system, in sensor system, and in power transmission system, because the chaotic behavior can destroy the stable operation of system. In fact, chaos control is usually a prerequisite for many practical applications in chaos engineering, such as permanent magnet synchronous motor [6], complex networks control [7], authenticated encryption [6], and chaos communications [8]. So, chaos control is of great practical significance and has received great interest due to its potential application in many fields in the recent years. Up to now, many control schemes have been proposed, like OGY scheme [9], OPF scheme [10], backstepping scheme [11], and so on [12-14].

On the other hand, the stability of linear fractionalorder system can be easily proved by the method proposed by Matignon [15]. However, this method can not be used to deal with the nonlinear fractional-order system. Based on Matignon's method [15], the local stability result for the nonlinear fractional-order system is proposed by Ahmed et al. [16]. In Ahmed's method [16], to evaluate the local stability of the equilibrium points in nonlinear fractionalorder system, the nonlinear system is converted to a linear system by linear approximation near the equilibrium point. Now, many researchers always used the Ahmed method to control the nonlinear fractional-order chaotic systems [1719]. But, it is known that the nonlinear problems of fractionalorder systems can not be straightforward changed to the linear problems. So, this local stability [16] maybe needs to be further studied.

Motivated by what is mentioned above, based on the Mittag-Leffler function in two parameters and Gronwall's Lemma, a modified fractional-order Chua's chaotic circuit can be stable via a fractional-order scalar controller, which can be determined by the Caputo fractional derivative of a single input. Ahmed's method [16] is not used in our paper. The numerical simulations are given and show the feasibility of the control scheme.

This paper is organized as follows. In Section 2, a modified fractional-order Chua's chaotic circuit is reported, and the chaotic attractor is given. In Section 3, two control schemes are proposed to stabilize this fractional-order Chua's chaotic circuit, and the numerical simulations are given. Conclusion is given in Section 4. 


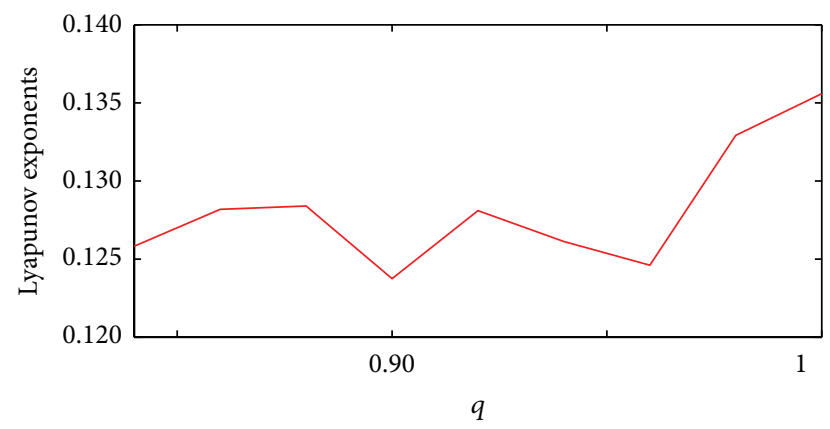

FIgure 1: The LLE of fractional-order Chua's circuit (1) varies as fractional-order $q$.

\section{A Modified Fractional-Order Chua's Chaotic Circuit}

Chua's circuit [20] was the physical chaotic system discovered by Chua et al. in 1986. Since then, Chua's circuit has been chosen as a classical paradigm in the research of chaos. Now, some modified Chua's circuits including their fractionalorder model have been reported by many researchers. In 2010, Muthuswamy and Chua [21] reported a simplest and autonomous modified Chua's chaotic circuit which includes only three elements in series. Based on [21], a modified Chua's circuit with fractional order is given in this paper, and its mathematical model is shown as follows:

$$
\begin{aligned}
& { }_{0}^{c} D_{t}^{q} x_{1}=x_{2}, \\
& { }_{0}^{c} D_{t}^{q} x_{2}=-\frac{x_{1}}{2}+\frac{x_{2}}{2}-\frac{x_{3}^{2} x_{2}}{2}, \\
& { }_{0}^{c} D_{t}^{q} x_{3}=-x_{2}-1.2 x_{3}+x_{2} x_{3},
\end{aligned}
$$

where $q=0.98$ is the fractional order and ${ }_{0}^{c} D_{t}^{q} x_{i}=(1 / \Gamma(n-$ q)) $\int_{0}^{t}\left(x_{i}^{(n)}(\tau) d \tau /(t-\tau)^{q+1-n}\right)(i=1,2,3)$ is the Caputo fractional derivative for state variable $x_{i}(i=1,2,3)$. Here $n-1<q<n, n$ is the smallest integer larger than $q, \Gamma(n-q)=$ $\int_{0}^{+\infty} t^{(n-q-1)} e^{-t} d t$, and $x_{i}^{(n)}(t)$ is the $n$th derivative in the usual sense.

By numerical calculation [22], we can obtain that the largest Lyapunov exponent (LLE) of fractional-order Chua's circuit (1) is larger than zero for $0.84 \leq q \leq 1$. Conversely, for $0<q<0.84$, the LLE is smaller than zero. The LLE of fractional-order Chua's circuit (1) with respect to the fractional-order $q$ is shown in Figure 1. For example, the LLE is 0.1329 for $q=0.98$, and its chaotic attractor is shown as in Figure 2. The LLE is 0.1356 for $q=1$.

According to what is mentioned above, the fractionalorder Chua's circuit (1) is chaotic for $0.84 \leq q \leq 1$.

\section{Stabilization of the Fractional-Order Chua's Chaotic Circuit (1)}

In this section, how to stabilize the fractional-order Chua's chaotic circuit (1) will be discussed. First we report some preliminary results.
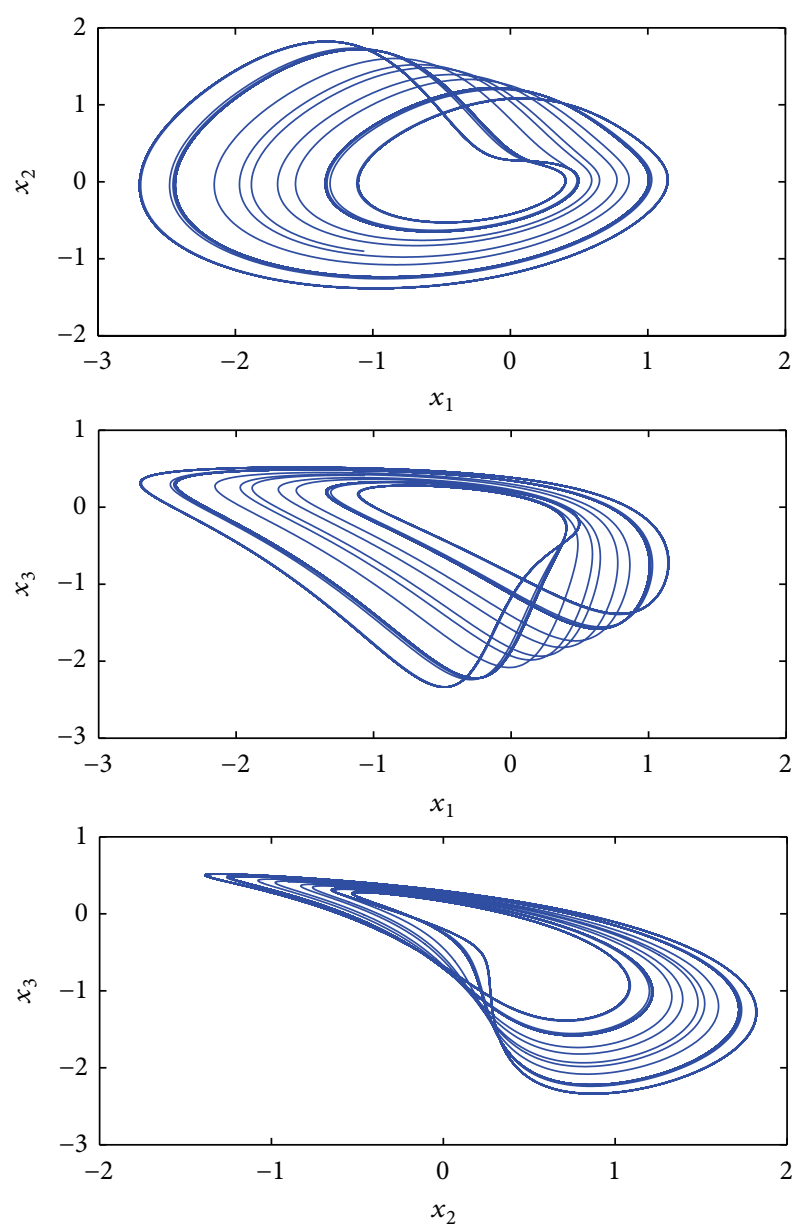

FIGURE 2: Chaotic attractor in the fractional-order Chua's circuit (1) for $q=0.98$.

Lemma 1 (see [23] Gronwall's Lemma). Let $a(t), b(t)$, and $c(t)$ be real-valued piecewise continuous functions, and let $c(t)$ be nonnegative in a real time interval $t \in\left[t_{1}, t_{2}\right]$. For all $t$ in $\left[t_{1}, t_{2}\right]$, if

$$
a(t) \leq b(t)+\int_{t_{1}}^{t_{2}} c(\vartheta) a(\vartheta) d \vartheta
$$

then

$$
a(t) \leq b(t)+\int_{t_{1}}^{t_{2}} c(\vartheta) a(\vartheta) \exp \left[\int_{\vartheta}^{t} c(v) d v\right] d \vartheta
$$

Lemma 2 (see [24] Wen et al., 2008). Consider a real matrix $K \in R^{n \times n}$, and let $\lambda_{i}(K)(i=1,2, \ldots, n)$ be its eigenvalues. If $q \pi / 2<\arg \lambda_{i}(K) \leq \pi$ holds, then

$$
\left\|M_{q, p}(K)\right\| \leq \rho(1+\|K\|)^{-1},
$$

where $\rho>0$ be a constant, $\|K\|$ is the $l_{2}$ norm for $K$, and $M_{q, p}(x)=\sum_{n=0}^{\infty}\left(x^{n} / \Gamma(q n+p)\right)(q>0, p>0)$ is the MittagLeffler function in two parameters $(p, q)$.

Now, we are in the position to state the main result of this paper. 
Theorem 3. Let the controlled system be

$$
\begin{aligned}
& { }_{0}^{c} D_{t}^{q} x_{1}=x_{2}+u\left(x_{2}\right), \\
& { }_{0}^{c} D_{t}^{q} x_{2}=-\frac{x_{1}}{2}+\frac{x_{2}}{2}-\frac{x_{3}^{2} x_{2}}{2}, \\
& { }_{0}^{c} D_{t}^{q} x_{3}=-x_{2}-1.2 x_{3}+x_{2} x_{3},
\end{aligned}
$$

where fractional-order $q=0.98$, let $u\left(x_{2}\right)=l_{1}\left({ }_{0}^{c} D_{t}^{q} x_{2}\right)$ be a fractional-order scalar controller determined by the Caputo derivative of the state variable $x_{2}$, and let $l_{1}$ be suitable feedback coefficient. If $1 \leq l_{1}<1+2 \sqrt{2}$, then $x_{i}(t)=0(i=1,2,3)(t \geq$ $\left.t_{0} \geq 0\right)$ is a stable solution of the nonlinear fractional-order system (5).

Proof. System (5) can be rewritten as

$$
{ }_{0}^{c} D_{t}^{q} x(t)=K x(t)+g(x(t)),
$$

where

$$
\begin{aligned}
g(x(t)) & =\left(\begin{array}{c}
-\frac{l_{1} x_{3}^{2}(t) x_{2}(t)}{2} \\
\frac{x_{3}^{2}(t) x_{2}(t)}{2} \\
x_{2}(t) x_{3}(t)
\end{array}\right), \\
K & =\left(\begin{array}{ccc}
-\frac{l_{1}}{2} & 1+\frac{l_{1}}{2} & 0 \\
-\frac{1}{2} & \frac{1}{2} & 0 \\
0 & -1 & -1.2
\end{array}\right) .
\end{aligned}
$$

Letting $\beta=q\|K\|$ be a constant, it is easy to obtain the following:

$$
\beta=q\|K\|>1
$$

According to system (6), it is easy to obtain that

$$
\begin{aligned}
& \left.g(x(t))\right|_{x(t)=0}=0 \\
& \quad \frac{\|g(x(t))\|}{\|x(t)\|} \leq\left|x_{3}(t)\right| \sqrt{1+\frac{\left(1+l_{1}^{2}\right) x_{3}^{2}(t)}{4}}
\end{aligned}
$$

hence

$$
\lim _{x(t) \rightarrow 0} \frac{\|g(x(t))\|}{\|x(t)\|}=0 .
$$

According to (9)-(11), there exist a constant $\rho>0$ and $\delta>0$ such that

$$
\|g(x(t))\|<\rho^{-1}\|x(t)\|
$$

for $\|x(t)\|<\delta$ and $t \geq 0$.
According to the controlled system (6), one can obtain the eigenvalues of matrix $K$ as follows:

$$
\begin{aligned}
& \lambda_{1}=-1.2 \\
& \lambda_{ \pm}=0.5\left(\frac{1}{2}-\frac{l_{1}}{2}\right) \pm 0.5 \sqrt{\left(\frac{1}{2}-\frac{l_{1}}{2}\right)^{2}-2} .
\end{aligned}
$$

Because $1 \leq l_{1}<1+2 \sqrt{2}$, one has

$$
\left|\arg \lambda_{ \pm}(K)\right|>\frac{\pi}{2}
$$

According to (13)-(15), we have

$$
\begin{aligned}
\left|\arg \lambda_{i}(K)\right|>\frac{\pi q}{2}, \quad(i=1,2,3), \\
\left|\arg \lambda_{i}\left(t^{q} K\right)\right|>\frac{\pi q}{2}, \quad(i=1,2,3) .
\end{aligned}
$$

Next, we study solution $x(t)$ in the fractional-order system (6). Now, one can take Laplace transform $L[\cdot]$ on system (6) and has

$$
s^{q} L[x(t)]-s^{q-1} x(0)=K L[x(t)]+L[g(x(t))],
$$

where $x(0)$ is the initial condition. So one has

$$
L[x(t)]=\frac{s^{q-1} x(0)}{\left(s^{q}-K\right)}+\frac{L[g(x(t))]}{\left(s^{q}-K\right)} .
$$

Meanwhile, one can take Laplace inverse transform for (19) and obtain

$$
\begin{aligned}
x(t)= & M_{q, 1}\left(K t^{q}\right) x(0) \\
& +\int_{0}^{t}(t-\tau)^{q-1} M_{q, q}\left(K(t-\tau)^{q}\right) g(x(\tau)) d \tau .
\end{aligned}
$$

Give $\delta_{0}\left(0<\delta_{0}<\delta\right)$ arbitrarily small, and consider solution $x(t)$ in system (6) with initial condition $\|x(0)\|<\delta_{0}$. Using formulas (4), (8), (12), and (17), (20) gives

$$
\begin{aligned}
& \|x(t)\| \\
& \quad \leq \rho \delta_{0}\left[1+\beta q^{-1} t^{q}\right]^{-1} \\
& \quad+\int_{0}^{t}(t-\tau)^{q-1}\left[1+\beta q^{-1}(t-\tau)^{q}\right]^{-1}\|x(\tau)\| d \tau .
\end{aligned}
$$

Using Lemma 1, inequality (21) can be changed as

$$
\begin{aligned}
\|x(t)\| \leq & \rho \delta_{0}\left[1+\beta q^{-1} t^{q}\right]^{-1} \\
& +\int_{0}^{t} \frac{\rho \delta_{0}(t-\tau)^{q-1}\left(1+\beta q^{-1} \tau^{q}\right)^{-1}}{\left[1+\beta q^{-1}(t-\tau)^{q}\right]^{1-\beta^{-1}}} d \tau .
\end{aligned}
$$




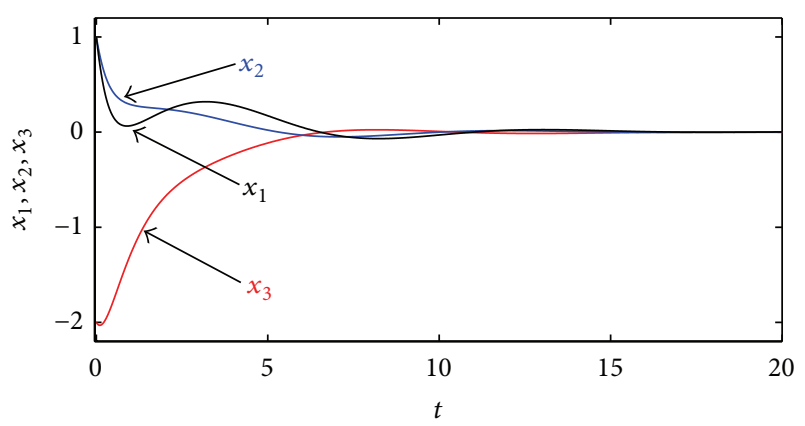

FIGURE 3: Control results for fractional-order system (5).

Due to $t-\tau \geq \tau$ in time interval $\tau \in[0, t / 2]$ and $t-\tau \leq \tau$ in time interval $\tau \in[t / 2, t]$, so

$$
\begin{aligned}
& \int_{0}^{t} \frac{(t-\tau)^{q-1}\left(1+\beta q^{-1} \tau^{q}\right)^{-1}}{\left[1+\beta q^{-1}(t-\tau)^{q}\right]^{1-\beta^{-1}}} d \tau \\
& \leq \int_{0}^{t / 2} \frac{\tau^{q-1}\left(1+\beta q^{-1} \tau^{q}\right)^{-1}}{\left[1+\beta q^{-1} \tau^{q}\right]^{1-\beta^{-1}}} d \tau \\
& \quad+\int_{t / 2}^{t} \frac{(t-\tau)^{q-1}\left[1+\beta q^{-1}(t-\tau)^{q}\right]^{-1}}{\left[1+\beta q^{-1}(t-\tau)^{q}\right]^{1-\beta^{-1}}} d \tau \\
& \left.\left.=2 \int_{0}^{t / 2} \frac{\tau^{q-1}\left(1+\beta q^{-1} \tau^{q}\right)^{-1}}{\left[1+\beta q^{-1} \tau^{q}\right]^{1-\beta^{-1}} d \tau} d q^{-1}\left(\frac{t}{2}\right)^{q}\right]^{\beta^{-1}-1}-1\right\} \\
& =\frac{2}{1-\beta}\left\{\left[1+\beta q^{-\beta}\right.\right.
\end{aligned}
$$

By (23), inequality (22) can be changed as follows:

$$
\begin{aligned}
& \|x(t)\| \\
& \leq \rho \delta_{0}\left[1+\beta q^{-1} t^{q}\right]^{-1} \\
& \quad+\frac{2 \rho \delta_{0}}{1-\beta}\left\{\left[1+\beta q^{-1}\left(\frac{t}{2}\right)^{q}\right]^{\beta^{-1}-1}-1\right\} \\
& <\rho \delta_{0}\left\{\frac{2}{\beta-1}+\frac{q}{\beta} t^{-q}-\frac{2 q}{\beta(\beta-1)}\left(1+\left(\frac{t}{2}\right)^{q}\right)^{-1}\right\} .
\end{aligned}
$$

Since $\delta_{0}$ is arbitrarily small, when time is long enough, based on inequality (24), one can obtain that $x_{i}(t)=0(i=$ $1,2,3)$ is a stable solution of the controlled system (5), which allows concluding the proof.

For example, let the initial conditions be $\left(x_{1}(0), x_{2}(0)\right.$, $\left.x_{3}(0)\right)=(1,1,-2)$; the simulation result is shown as in Figure 3 with $l_{1}=2$.

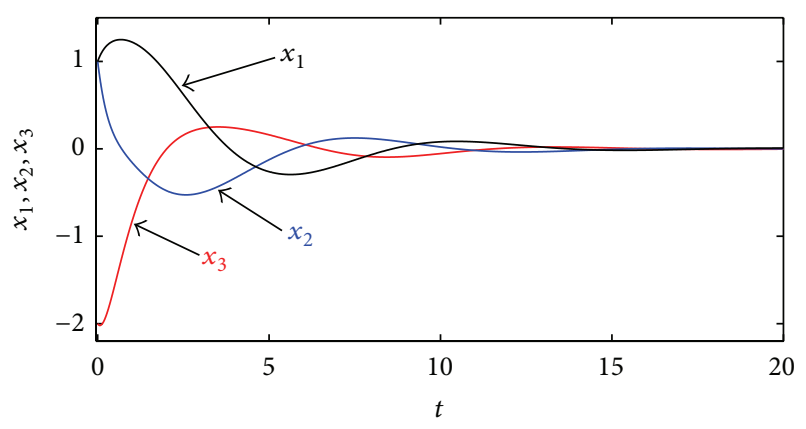

Figure 4: Control results for fractional-order system (25).

Theorem 4. Let the controlled system be

$$
\begin{aligned}
& { }_{0}^{c} D_{t}^{q} x_{1}=x_{2}, \\
& { }_{0}^{c} D_{t}^{q} x_{2}=-\frac{x_{1}}{2}+\frac{x_{2}}{2}-\frac{x_{3}^{2} x_{2}}{2}+u\left(x_{1}\right), \\
& { }_{0}^{c} D_{t}^{q} x_{3}=-x_{2}-1.2 x_{3}+x_{2} x_{3},
\end{aligned}
$$

where fractional-order $q=0.98$, let $u\left(x_{1}\right)=l_{1}\left({ }_{0}^{c} D_{t}^{q} x_{1}\right)$ be a fractional-order scalar controller determined by the Caputo derivative of the state variable $x_{1}$, and let $l_{1}$ be suitable feedback coefficient. If $-1 / 2-\sqrt{2} \leq l_{1}<-1 / 2$, then $x_{i}(t)=0(i=1,2,3)$ is a stable solution of system (25).

Proof. System (25) can be rewritten as

$$
{ }_{0}^{c} D_{t}^{q} x(t)=K x(t)+g(x(t)),
$$

where

$$
\begin{aligned}
g(x(t)) & =\left(\begin{array}{c}
0 \\
\frac{x_{3}^{2}(t) x_{2}(t)}{2} \\
x_{2}(t) x_{3}(t)
\end{array}\right), \\
K & =\left(\begin{array}{ccc}
0 & 1 & 0 \\
-\frac{1}{2} & l_{1}+\frac{1}{2} & 0 \\
0 & -1 & -1.2
\end{array}\right) .
\end{aligned}
$$

Now, we can complete the proof in a similar way with Theorem 3.

For example, let the initial conditions be $\left(x_{1}(0), x_{2}(0)\right.$, $\left.x_{3}(0)\right)=(1,1,-2)$; the simulation results are shown as in Figure 4 with $l_{1}=-1$.

\section{Conclusions}

Based on an autonomous 3D Chua's chaotic circuit, a modified fractional-order Chua's circuit system is proposed. By numerical calculation, we obtain that the largest Lyapunov exponent of the modified fractional-order Chua's circuit 
system is larger than zero for $0.84 \leq q \leq 1$. This result indicates that the modified fractional-order Chua's circuit system exhibits chaotic behavior if $0.84 \leq q \leq 1$. For example, the largest Lyapunov exponent of the modified fractionalorder Chua's circuit system is 0.1329 for $q=0.98$. The chaotic attractor for $q=0.98$ is given. By using Laplace transform, Gronwall's Lemma, and Mittag-Leffler function in two parameters, we proposed two control strategies to stabilize the modified fractional-order Chua's chaotic circuit. In our control scheme, a scalar controller determined by a single input and its Caputo derivative is used. The numerical simulations show the proposed control strategies are valid.

\section{Conflict of Interests}

The authors declare that there is no conflict of interests regarding the publication of this paper.

\section{References}

[1] L. O. Chua, "Memristor-the missing circuit element," IEEE Transactions Circuit Theory, vol. 18, no. 5, pp. 507-519, 1971.

[2] T. T. Hartley, C. F. Lorenzo, and H. K. Qammer, "Chaos in a fractional order Chua's system," IEEE Transactions on Circuits and Systems I: Fundamental Theory and Applications, vol. 42, no. 8, pp. 485-490, 1995.

[3] D. Q. Wei, L. Wan, X. S. Luo, S. Y. Zeng, and B. Zhang, "Global exponential stabilization for chaotic brushless DC motors with a single input," Nonlinear Dynamics, vol. 77, no. 1-2, pp. 209-212, 2014.

[4] P. Palevicius, M. Ragulskis, A. Palevicius, and V. Ostasevicius, "Applicability of time-averaged holography for micro-electromechanical system performing non-linear oscillations," Sensors, vol. 14, no. 1, pp. 1805-1821, 2014.

[5] M. P. Aghababa and H. P. Aghababa, "The rich dynamics of fractional-order gyros applying a fractional controller," Proceedings of the Institution of Mechanical Engineers. Part I, vol. 227, no. 7, pp. 588-601, 2013.

[6] Q. Wei, X.-Y. Wang, and X.-P. Hu, "Optimal control for permanent magnet synchronous motor," Journal of Vibration and Control, vol. 20, no. 8, pp. 1176-1184, 2014.

[7] L. P. Chen, J. F. Qu, Y. Chai, R. Wu, and G. Qi, "Synchronization of a class of fractional-order chaotic neural networks," Entropy, vol. 15, no. 8, pp. 3265-3276, 2013.

[8] P. Louodop, H. Fotsin, S. Bowong, and A. Soup Tewa Kammogne, "Adaptive time-delay synchronization of chaotic systems with uncertainties using a nonlinear feedback coupling," Journal of Vibration and Control, vol. 20, no. 6, pp. 815-826, 2014.

[9] E. Ott, C. Grebogi, and J. A. Yorke, "Controlling chaos," Physical Review Letters, vol. 64, no. 11, pp. 1196-1199, 1990.

[10] N. Inaba and T. Nitanai, "OPF chaos control in a circuit containing a feedback voltage pulse generator," IEEE Transactions on Circuits System I-Fundamental Theory Applications, vol. 45, no. 4, pp. 473-480, 1998.

[11] S. Mascolo and G. Grassi, "Controlling chaotic dynamics using backstepping design with application to the Lorenz system and Chua's circuit," International Journal of Bifurcation and Chaos in Applied Sciences and Engineering, vol. 9, no. 7, pp. 1425-1434, 1999.
[12] T.-B. T. Nguyen, T.-L. Liao, and J.-J. Yan, "Adaptive tracking control for an uncertain chaotic permanent magnet synchronous motor based on fuzzy neural networks," Journal of Vibration and Control, vol. 21, no. 3, pp. 580-590, 2015.

[13] Y. Xu, H. Wang, D. Liu, and H. Huang, "Sliding mode control of a class of fractional chaotic systems in the presence of parameter perturbations," Journal of Vibration and Control, vol. 21, no. 3, pp. 435-448, 2015.

[14] Q. Wei and X.-Y. Wang, "Chaos controlling of permanent magnet synchronous motor base on dither signal," Journal of Vibration and Control, vol. 19, no. 16, pp. 2541-2550, 2013.

[15] D. Matignon, "Stability results for fractional differential equations with applications to control processing," in Proceedings of the International IEEE-SMC Multiconference on Computational Engineering in Systems Applications (IMACS '96), vol. 2, pp. 963-968, Lille, France, 1996.

[16] E. Ahmed, A. M. El-Sayed, and H. A. A. El-Saka, "Equilibrium points, stability and numerical solutions of fractional-order predator-prey and rabies models," Journal of Mathematical Analysis and Applications, vol. 325, no. 1, pp. 542-553, 2007.

[17] P. Zhou, R. Ding, and Y.-X. Cao, "Multi drive-one response synchronization for fractional-order chaotic systems," Nonlinear Dynamics, vol. 70, no. 2, pp. 1263-1271, 2012.

[18] Z. Odibat, "A note on phase synchronization in coupled chaotic fractional order systems," Nonlinear Analysis: Real World Applications, vol. 13, no. 2, pp. 779-789, 2012.

[19] A. S. Hegazi, E. Ahmed, and A. E. Matouk, "On chaos control and synchronization of the commensurate fractional order Liu system," Communications in Nonlinear Science and Numerical Simulation, vol. 18, no. 5, pp. 1193-1202, 2013.

[20] L. O. Chua, M. Komuro, and T. Matsumoto, "The double scroll family," IEEE transactions on circuits and systems, vol. 33, no. 11, pp. 1072-1118, 1986.

[21] B. Muthuswamy and L. O. Chua, "Simplest chaotic circuit," International Journal of Bifurcation and Chaos, vol. 20, no. 5, pp. 1567-8150, 2010.

[22] C. P. Li and G. J. Peng, "Chaos in Chen's system with a fractional order," Chaos, Solitons \& Fractals, vol. 22, no. 2, pp. 443-450, 2004.

[23] G. S. Jones, "Fundamental inequalities for discrete and discontinuous functional equations," Journal of the Society for Industrial and Applied Mathematics, vol. 12, no. 1, pp. 43-57, 1964.

[24] X.-J. Wen, Z.-M. Wu, and J.-G. Lu, "Stability analysis of a class of nonlinear fractional-order systems," IEEE Transactions on Circuits and Systems II: Express Briefs, vol. 55, no. 11, pp. 1178$1182,2008$. 


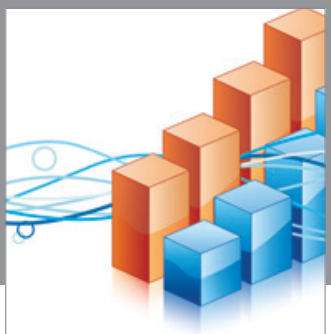

Advances in

Operations Research

vatem alat4

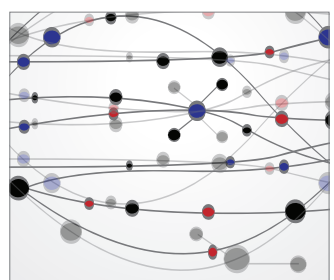

\section{The Scientific} World Journal
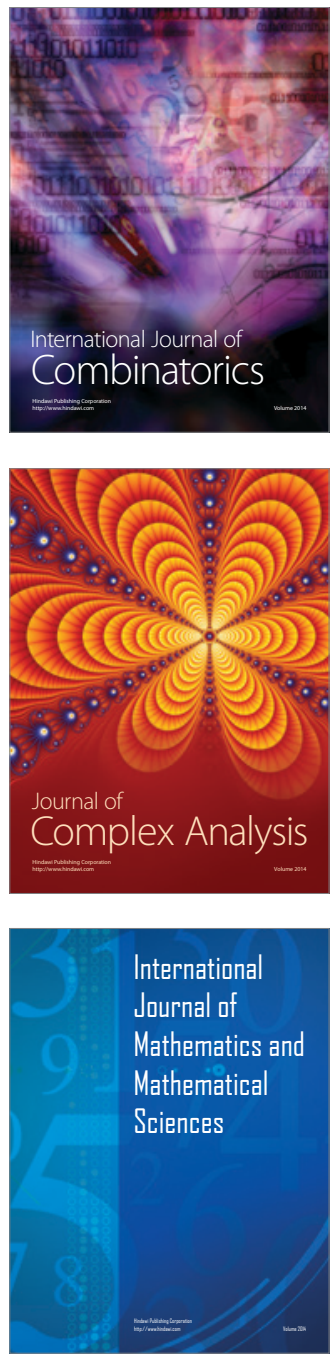
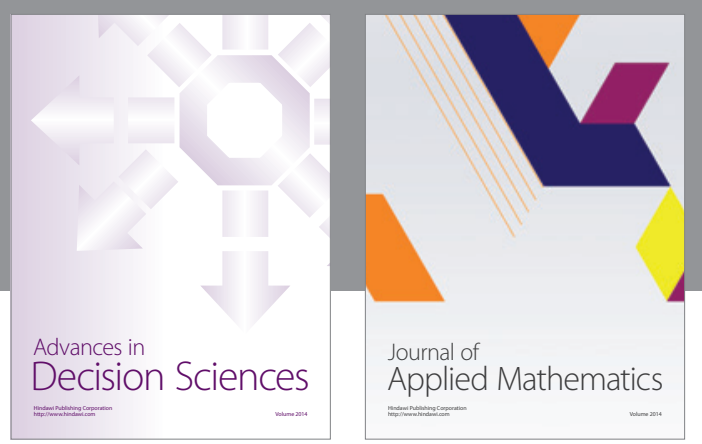

Algebra

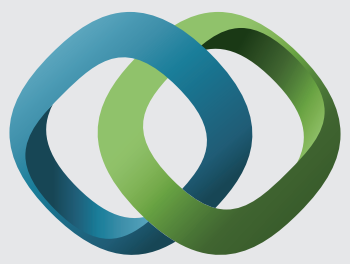

\section{Hindawi}

Submit your manuscripts at

http://www.hindawi.com
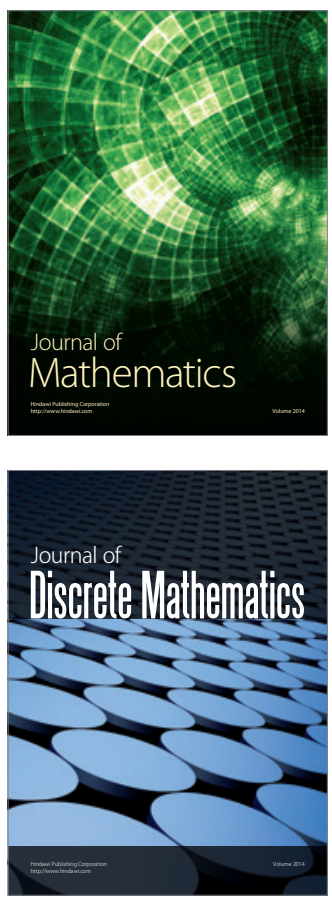

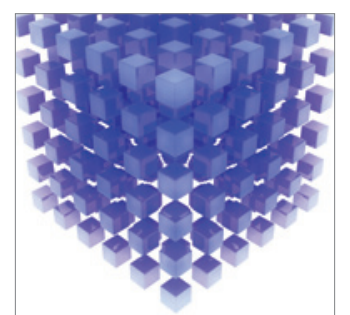

Mathematical Problems in Engineering
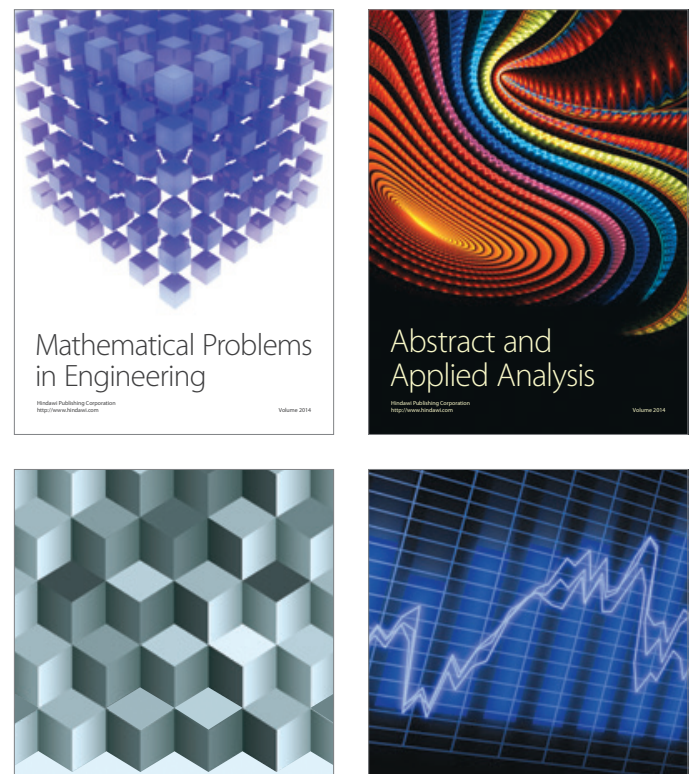

Journal of

Function Spaces

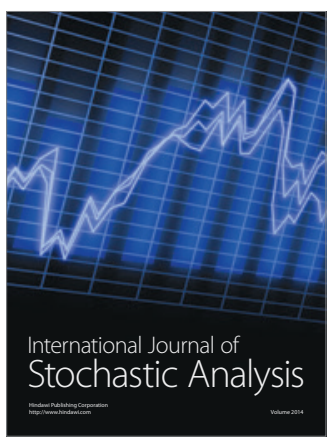

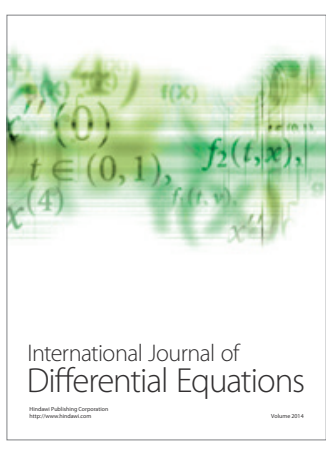
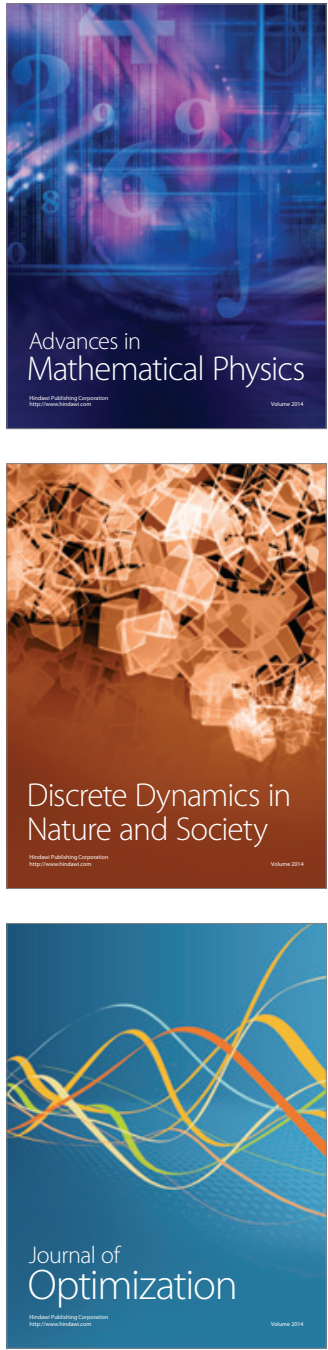